\title{
Características morfométricas de la red parafluvial de la comarca de la Manchuela (Albacete)
}

\author{
ANTONIO FERNÁNDEZ FERNÁNDEZ*
}

\begin{abstract}
RESUMEN SUMMARY
El valle del Júcar a su paso por el The Jucar river valley in the northern norte de la provincia de Albacete, en el of the Albacete provirce, has a parafluvial system (dry valleys). borde suroriental de la Submeseta sur, se caracteriza por la presencia de una red parafluvial. Ésta drena al Júcar, de Sometimes tipical atmospherics situations associated at the manera ocasional, importantes descargas de caudal coincidiendo con mediterranean sea, are the origin of strongs precipitations. This high intensas precipitaciones asociadas a situaciones atmosféricas de "gota fria", características del ámbito mediterráneo. Las diferentes ramblas del sector presentan una morfometría muy condicionada por la disposición del relieve y la heterogeneidad precipitations made overfloods and generate importants catastrophic situations. The dry valleys denominated ramblas in the area presents a morphometry conditionated by the litology ad orographic aspects. litológica, este aspecto es el analizado en el presente trabajo.
\end{abstract}

\section{INTRODUCCIÓN}

El valle del Júcar en su tramo medio que recorre el norte de la provincia de Albacete se caracteriza por una marcada ausencia de tributarios con circulación de agua permanente. La red de afluentes está constituida

* Dpto. de Geografía. UNED. 
por un denso conjunto de aparatos parafluviales de funcionamiento intermitente y esporádico, mal alimentados y que sólo canalizan agua en situaciones de fuertes precipitaciones. Desde el punto de vista geomorfológico estos cursos se definen como ramblas y barrancos, cuya longitud y configuración son muy desiguales. La existencia de esta particular tipología de drenaje está determinada por tres factores interdependientes: las características climáticas e hidrológicas del área, el control estructural y el estado de la cubierta vegetal.

El clima se caracteriza por una acusada irregularidad y estacionalidad de las precipitaciones y, sobre todo, por la concentración de los aportes pluviométricos en cortos e intensos aguaceros, que dan lugar a unos violentos y repentinos procesos de escorrentía superficial, cuyos caudales son jerarquizados por una red de canales muy diferentes, morfológica y funcionalmente, de los presentados por los lechos fluviales. Esta modalidad climático-hidrológica es propia y característica de las áreas próximas al litoral mediterráneo, que incluso deja sentir su influencia en el sector más oriental de la meseta, donde se encuadra la fosa del Júcar.

La variedad de los materiales sedimentarios que constituyen la estructura geológica de dicha cuenca, por su parte, influye de forma significativa en la diversificada configuración de estos cursos parafluviales, cuyos parámetros morfométricos (índice de sinuosidad, grado de jerarquización, aspecto del fondo, perfil longitudinal etc.) varían dependiendo del dominio morfolitológico que atraviesen.

Finalmente, el débil recubrimiento vegetal de las laderas a causa de la escasez pluviométrica y de la acción antrópica, facilita los procesos erosivos y posibilita el peculiar funcionamiento de la escorrentía superficial de las aguas, del que resultan los caracteres geomorfológicos de las ramblas.

Estos afluentes esporádicos del Júcar permanecen inactivos durante dilatados períodos de tiempo, sin embargo, su funcionamiento breve y ocasional, posee una elevada energía morfogenética, tanto por su capacidad de incisión, como de arrastre y sedimentación de materiales. También son importantes indicadores paleoambientales, al quedar registrados en los fondos y aterrazamientos de sus lechos las diferentes fases de actividad.

\section{DEFINICIÓN DE RAMBLA Y BARRANCO}

No existe una definición de término "rambla" aceptada unánimemente por los geomorfólogos, aunque han sido numerosos los autores que han tratado el problema, destacando entre otros (Vilá Valentí, J., 1961), (Mateu 
Belles, J.F.,1982) (Pérez Cueva, A., 1985) y (Segura, F., 1988). Todos ellos coinciden en definir una ausencia de corriente permanente de agua, pero difieren en la explicación de la génesis de los canales y en la definición del umbral de días necesarios para acotar el concepto de "caudales esporádicos", así como en diversos aspectos morfométricos y cuantitativos. A la imprecisión dentro del campo científico hay que añadir la complejidad toponimica con referencia a estos lechos secos la mayor parte del año.

En el área de estudio se utilizan los siguientes términos para referirse a ellos: rambla, ramblilla, ramblazo, cañada, cañizo, cañadilla, cañejo, arroyo, vallejo, barranco y barranquera. Esta diversificada nomenclatura no tiene un significado hidrogeomorfológico preciso, de tal manera que los términos barranco, rambla y cañada pueden utilizarse indistintamente para designar cauces de características similares. El análisis de la toponimia local no aporta por tanto, criterios eficaces a la hora de delimitar lo que se entiende por rambla en el presente trabajo.

El estudio de la bibliografía y la observación detallada del territorio nos ha llevado a adoptar el criterio siguiente: se entenderá por rambla a todo aquel sistema de cauces que muestren una jerarquía organizativa de orden 20 superior, según la metodología propuesta por (Strahler, A., 1964) y que carezca de caudal diario y contínuo en su canal principal. De acuerdo con esta descripción se dispone de un método sencillo de definición, que permite también establecer cuál es el canal principal del sistema (que coincidirá siempre con el segmento de mayor orden). De este modo se puede conocer con exactitud la longitud y la altura máxima de dicho canal principal. La anchura del fondo y el volumen de los caudales de las ramblas no se mostraron como variables útiles por la escasa significación metodológica de la primera y la ausencia de datos de aforo referentes al segundo.

La palabra barranco quedaría para aquellos casos en los que el canal principal constituye un único eje. Generalmente éstos presentan una pendiente longitudinal más elevada, un recorrido más corto y una mayor profundidad respecto a su anchura que las ramblas.

Aplicando el criterio anteriormente expuesto, se han descrito nueve ramblas, que confluyen al Júcar por su margen derecha excepto una que lo hace por la izquierda. Esta disimetría está en relación con la configuración del relieve en la fosa del Júcar.

\section{DESCRIPCIÓN DE LAS RAMBLAS}

Las nueve ramblas definidas se pueden agrupar, por sus características comunes, en tres conjuntos. 


\section{A) RAMBLAS QUE TIENEN SUS CABECERAS EN UN ÁREA LLANA.}

Tienen su nacimiento en la llanura de La Mancha o en La Manchuela. Se caracterizan por una escasa pendiente en los tramos de cabecera y un trazado meandriforme y divagante en la llanura. A este grupo corresponden las ramblas de Cañahorro y Abengibre.

\section{Rambla de Cañahorro.}

Los cauces de primer orden que la forman nacen en la llanura de la Manchuela, en el sector comprendido entre las poblaciones de Valdeganga y Casas de Juan Núñez; el canal principal desemboca en la margen derecha del Júcar en las proximidades de Los Malecones, a medio camino entre las poblaciones de Valdeganga y Maldonado.

\section{Rambla de Abengibre.}

Su nacimiento se sitúa fuera de los dominios morfoestructurales de la Manchuela, ya que las primeras cabeceras se localizan en las proximidades de Motilla del Palancar, a 50-60 km. de su confluencia con el Júcar, en la población de Jorquera. Esta rambla es la única que alcanza al río procedente del norte.

B) RAMBLAS QUE NACEN EN LA MUELA DE CARCELÉN.

Este grupo estaría constituido por ramblas cuyas cabeceras arrancan de los relieves cretácicos situados al sur, fuera del dominio morfoestructural de la Manchuela. Comparte como características comunes largos recorridos y redes muy densas y complejas, desembocando en el tramo del río comprendido entre La Recueja y Alcalá.

\section{Rambla de Ayora o La Recueja.}

Los diversos cauces que conforman su cabecera arrancan de relieves montañosos situados 25-30 kilómetros al sur, correspondientes a las sierras de Chinchilla. La confluencia con el Júcar se realiza en la villa de La Recueja.

\section{Rambla de San Lorenzo.}

Al igual que la de Ayora, tiene sus múltiples cabeceras en los relieves de Chinchilla y, tras atravesar la fosa de Carcelén, va a buscar al Júcar confluyendo también en el tramo desarrollado entre La Recueja y Alcalá, exactamente en el lugar donde se emplaza la Ermita de San Lorenzo, que da el nombre a la rambla.

C) RAMBLAS QUE NACEN EN LA SIERRA DE LA CABALLA.

Este tercer grupo está constituído por las ramblas que desembocan en el tramo inferior del río, desde Alcalá a la presa del Molinar. Tienen como 
caracteristicas comunes su corto recorrido y su fuerte desnivel. Todas ellas nacen en las estribaciones de los relieves cretácicos de La Caballa, atraviesan en un breve trayecto la llanura de la Manchuela y parecen despeñarse en los rebordes de la hoz, buscando el nivel de base que marca el Júcar. Este conjunto, que es el más numeroso, está constituido por cinco ramblas.

\section{Rambla de Alcalá.}

Los cauces de orden 1 que la dan origen arrancan pocos kilómetros al sur de Alcalá del Júcar por lo que su recorrido es corto. Su desembocadura se realiza en la citada población encontrándose urbanizado el propio fondo de la rambla, a pesar del riesgo que ello conlleva.

\section{Rambla del Cura.}

Su recorrido es muy corto y presenta un fuerte desnivel entre el nacimiento y la desembocadura que se realiza aguas abajo de la aldea de Tolosa.

\section{Rambla de Casas del Conde.}

Su nacimiento se localiza pocos kilómetros al este del de la anterior. Su canal principal pasa por las cercanías del caserío de Casas del Conde, para buscar la confluencia del Júcar en el tramo comprendido entre la central eléctrica de El Bosque y el paraje denominado el Tranco del Lobo.

\section{Barranco de Mingo.}

Aunque la toponimia no le dé el nombre de rambla, se considera como tal al cumplir el criterio metodológico ya señalado, si bien, se mantiene su denominación local. Sus características son similares a las anteriores ramblas de su grupo. Su desembocadura en el Júcar se realiza aguas abajo del Tranco del Lobo.

\section{Rambla del Molinar.}

Es la más oriental y su cabecera, al igual que las de este grupo, se instala en los materiales cretácicos de la sierra de La Caballa y la desembocadura se realiza en la cola del embalse del Molinar. Además de estas ramblas, la red hidrográfica del Júcar incluye una serie innumerable de barrancos, de corto recorrido y ausencia de jerarquización.

\section{METODOLOGIAA DEL ANÁLISIS MORFOMÉTRICO DE LAS RAMBLAS}

La metodología y los parámetros morfométricos escogidos para el análisis de la red parafluvial han sido los siguientes: 
Se ha dibujado la red a escala $1 / 50.000$, excepto la rambla de Abengibre que lo ha sido a $1 / 100.000$, dada su mayor longitud. La base cartográfica ha sido el M.T.N, del que se han obtenido los cauces en él expresamente representados, y se han añadido los canales inferidos a partir del dibujo de las curvas de nivel. Esta labor ha sido apoyada con la utilización de la fotografía aérea.

Una vez dibujada la red, se ha realizado su jerarquización siguiendo el método propuesto por (Strahler, A.,1964) y se ha hecho un recuento de los cauces según su respectivo orden. Éste ha atendido tanto al número de ellos existente dentro de cada orden como a los kilómetros que dichos cauces suman.

Se considera canal principal de la rambla o barranco aquel que registra el orden máximo, entendiendo que su inicio se encuentra en el punto de confluencia de los dos canales de orden inmediatamente inferior; la desembocadura del canal en el rio marca el final del segmento. Se ha obviado el criterio toponímico para establecer el inicio y la longitud del canal, por no coincidir con el criterio morfométrico. De este canal principal de la rambla se anota la altura máxima (punto de mayor altura en su recorrido), la longitud y la altura mínima (coincidente con su confluencia en el Júcar). Con estos tres parámetros se puede extraer el valor de la pendiente media de su recorrido, aunque ésta tiene importantes cambios dependiendo del substrato litológico. Del canal principal también se analiza el Índice de Sinuosidad, calculado al dividir la longitud del canal entre la distancia en línea recta que separa sus puntos extremos (Mueller, C.,1968).

Otros parámetros morfométricos son los relativos a la cuenca drenada por la rambla. Se ha medido la superficie en kilómetros cuadrados de ésta, así como su altura máxima y mínima. La altura máxima coincide generalmente con un relieve de la divisoria de aguas, pudiendo servir la misma cota como punto de referencia máximo a diversas cuencas. La altura minima de la cuenca, por su parte, coincide con la del canal principal. Dividiendo la longitud total de cada red entre la superficie de su cuenca se obtiene la Densidad de Drenaje. Cuanto mayor sea su valor la red de cursos temporales se considera más evolucionada y más eficaz a la hora de drenar la escorrentia superficial.

Dividiendo la superficie de la cuenca entre el número de cauces de orden 1 se obtiene el denominado Coeficiente de Torrencialidad, considerando que cuanto menor sea la superficie abarcada por unidad de cauce la potencialidad erosiva es mayor. Por último, se relaciona cada orden con el inmediatamente superior para averiguar la Razón de Bifurcación de Horton, según la cual un valor inferior a 2 indica cuencas de escaso relieve, los 
valores intermedios (de 3 a 5) corresponden a cuencas de montaña sobre roquedos de naturaleza lítica homogénea y los valores superiores a 5 indican cuencas significativamente afectadas por controles estructurales.

Con todos los datos descritos se ha confeccionado la siguiente ficha, que se ha cumplimentado para todas las ramblas estudiadas.

\begin{tabular}{llll}
\hline Orden & N. ${ }^{\circ}$ de cauces & Kilómetros & Longitud media \\
\hline
\end{tabular}

LONGITUD DEL CANAL PRINCIPAL

INDICE DE SINUOSIDAD DEL CANAL PRINCIPAL

ALTURA MÁXIMA DEL CANAL PRINCIPAL

ALTURA MINIMA DEL CANAL PRINCIPAL

PENDIENTE MEDIA DEL CANAL PRINCIPAL.

SUPERFICIE DE LA CUENCA

ALTURA MÁXIMA DE LA CUENCA

ALTURA MINIMA DE LA CUENCA.

DESNIVEL MÁXIMO DE LA CUENCA

DENSIDAD DE DRENAJE

INDICE DE TORRENCIALIDAD

RAZÓN DE BIFURCACIÓN DE HORTON.

En el anexo estadístico se muestran los valores realizados en al análisis de cada una de las ramblas. En el siguiente apartado se realizará el comentario de las principales.

\section{DESCRIPCIÓN MORFOMETRICA DE LAS RAMBLAS}

\section{V.1. Análisis morfométrico de la rambla de Abengibre}

La rambla de Abengibre es la que presenta mayor longitud y drena una mayor superficie; también es la única de las estudiadas que afluye al Júcar por su margen izquierda.

a) Topología y estructura de la red de drenaje.

La cuenca de la rambla de Abengibre tiene $670 \mathrm{~km}^{2}$ de superficie. De norte a sur alcanza $60 \mathrm{~km}$ de longitud y su anchura máxima es de unos 20 
kilómetros. Esta superficie es drenada por tres canales que, tras su conexión, darán lugar al eje de la rambla y que instalan sus cabeceras en la provincia de Cuenca, en un sector delimitado por los embalses de Alarcón y Contreras. Estos tres ejes son, de oeste a este, la Cañada del Hoyazo, la Cañada de La Vega y la Cañada de La Hoz.

La red de la rambla de Abengibre está constituída por canales muy largos pero escasamente dotados de tributarios lo cual, justifica el bajísimo valor de la Densidad de Drenaje $\left(0.39 \mathrm{~km} / \mathrm{km}^{2}\right)$ y un Índice de Torrencialidad de un cauce de primer orden por cada $14 \mathrm{~km}^{2}$ de cuenca, indicador de una débil potencialidad erosiva. Sin embargo, esta rambla se muestra muy agresiva en el aspecto erosivo y canaliza muy importantes caudales en los momentos de precipitación, debido a la gran superficie de su cuenca, lo que contrarresta con amplitud la baja potencialidad erosiva de su red y la escasa pendiente de sus cauces.

b) Características topográficas de la cuenca.

Pese a que el desnivel alcanza los $500 \mathrm{~m}$, las pendientes no son grandes debido a la gran superficie de la cuenca. Esto provoca que tanto los canales secundarios como la rambla de Abengibre, discurran ligeramente encajados en los terrenos calcáreos de la Ibérica y en los detríticos de La Mancha, con perfiles de escasa pendiente y de trazados de elevada sinuosidad.

c) Características del canal principal.

En sus 61 kilómetros de recorrido el canal principal de la rambla de Abengibre tiene un trazado muy meandriforme, como indica su índice de Sinuosidad de 1.74; valor que se incrementa en los últimos kilómetros de su recorrido, previos a la confluencia con el Júcar. Este trazado altamente sinuoso parece estar relacionado con un flujo divagante derivado de la escasa pendiente, inicialmente desarrollado sobre los materiales detríticos pliocenos de La Mancha-Manchuela y que después se ha fijado por sobreimposición en los estratos carbonatados subyacentes. El perfil longitu-

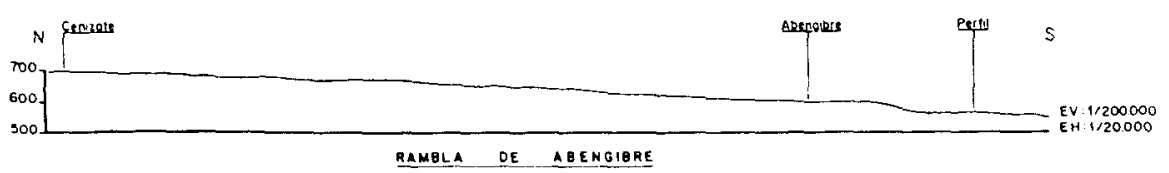

Figura 1. Perfil longitudinal de la rambla de Abengibre. 
dinal expuesto en la (figura 1) muestra el progresivo encajamiento que la rambla de Abengibre sufre en su tramo final.

Esta rambla muestra un fondo plano que soporta una fuerte explotación agrícola. En él son muy frecuentes los gaviones o "paradas" que, además de servir para delimitar las numerosas fincas, desempeñan la función de contrafuertes y muros de contención ante las posibles avenidas. La presencia y el mantenimiento de estas infraestructuras indican la potencialidad erosiva de la rambla en los momentos de avenida.

\section{V.2. Análisis morfométrico de la rambla de Ayora o La Recueja}

a) Topología y estructura de la red de drenaje.

A diferencia de la de Abengibre, la rambla de Ayora presenta una red compleja y estructurada con un número elevado de cauces de primer orden: 54. Su canal principal surge de la confluencia de dos canales de orden 3 , que tienen sus cabeceras en las estribaciones septentrionales de los montes de Chinchilla de Monte-Aragón y bordea el pueblo de Casas de Juan Núñez, en donde recibe el nombre de "Cañada de los Villares". A lo largo de su recorrido recibe por su margen derecha tres canales afluentes que organizan, cada uno de ellos, un subsistema de ramblas secundarias y barrancos. Estos tres canales afluentes de la rambla de Ayora o de la Recueja son de oeste a este: la Ramblilla del Tollo, el Vallejo de la Fuente de la Tía Juana y la Cañada de la Cardosa.

Estos tres ramales y el canal principal constituyen el esqueleto básico de la cuenca de la rambla de Ayora o de la Recueja, dentro de la cual el control estructural mediatiza el trazado y recorrido de los múltiples cauces de primer orden, como se detecta en el índice de Bifurcación de Horton, que es de 4.15 entre los cauces de orden 1 y 2 . Este valor encubre los hechos reales de que los barrancos discurren en ocasiones siguiendo ondulaciones sinclinales y que existe un claro eje estructural marcando el contacto entre los materiales plegados del cretácico de las sierras de Chinchilla y los detríticos que cubren la superficie de la fosa de Carcelén. En esta línea de contacto, la rambla de Ayora y los canales secundarios que drenan en ella adquieren trazados rectilíneos SSE-NNW y cursos paralelos.

El Índice de Horton entre los cauces de $2 .^{\circ}$ y $3 .^{\circ}$ orden, desciende a 2.6, indicativo de una rápida concentración en pocos canales de una escorrentía que hasta entonces habia sido canalizada en un elevado número de cauces. Este hecho, que implica un notable riesgo de inundación, 
coincide con la salida de las ramblas de orden 3 a la llanura y el abandono de los relieves montañosos.

Por último, el índice de bifurcación de Horton entre los canales de orden 3 y 4 es de 5 . Este elevado valor indica controles estructurales que, en este caso, parecen estar motivados por factores litológicos, como se expone en el siguiente punto $\mathrm{c}$.

b) Caracteristicas topográficas de la cuenca.

La superficie drenada por los casi 200 kilómetros de cauces de la red de la rambla de Ayora es de $198.45 \mathrm{~km}^{2}$. Esta cuenca alcanza una altura máxima de $1086 \mathrm{~m}$ en el pico de cerro del Buey (montes de Chinchilla), la cual contrasta con los $535 \mathrm{~m}$ de altura mínima en la confluencia con el Júcar, con un resultado de $551 \mathrm{~m}$ de desnivel. Éste indica una potencialidad erosiva relativamente importante, la cual queda matizada, sin embargo, por un escalonamiento topográfico altamente relacionado con los dominios litoestructurales. Se aprecian tres escalones topográficos, que son los siguientes:

- Uno primero correspondiente a los relieves plegados cretácicos de Chinchilla, en el que las alturas se sitúan entre los 860 y los $1086 \mathrm{~m}$.

- El segundo coincide con el recubrimiento detrítico de las fosas de Carcelén y de la Manchuela, cuya altura oscila entre 600 y $860 \mathrm{~m}$. Al tratarse de un sector de escaso desnivel y largo recorrido, los canales pierden en él pendiente facilitando el predominio de los procesos de sedimentación.

- El tercero es el referente a la entrada a la hoz de Júcar. De muy corto desarrollo, está comprendido entre los 600 y los $535 \mathrm{~m}$, lo que implica una intensificación de los procesos erosivos al aumentar espectacularmente el valor de la pendiente.

c) Litología y trazado de la red.

Los tres escalones o elementos hipsométricos que se han individualizado corresponden a otros tantos conjuntos litológicos bien diferenciados. El primero coincide con el afloramiento de los estratos calizos y dolomíticos del cretácico superior, cuya relativa dureza y disposición plegada hacen que el trazado de los cauces quede claramente orientado por la estructura. El segundo sector coincide, por su parte, con el recubrimiento detrítico de los glacis que arrancan desde los relieves cretácicos y cubren la superficie de la fosa de Carcelén y de parte de la Manchuela; en este tramo se ven facilitados los procesos de abandono de carga sedimentaria debido a la disminución de la pendiente, lo que se traduce en una ten- 
dencia a la colmatación de los fondos de los canales. El tercer ámbito altimétrico, donde se salvan las mayores pendientes (casi 200 metros en 1.5 ó 2 kilómetros de recorrido), se corresponde con el área en que el roquedo está constituido por los estratos de las calizas de Alcalá; el incremento de la inclinación favorece el encajamiento y la conservación de trazados meandrifromes de alta sinuosidad.

d) Características del canal principal.

La rambla de Ayora corta en su recorrido las tres unidades que se acaban de diferenciar en función de criterios altimétricos y lito-estructurales. Ello hace que la rambla presente una forma distinta en cada una de dichas unidades. (Figura 2).

En el perfil longitudinal es perceptible con claridad la acentuación de la pendiente en las inmediaciones de la confluencia con el Júcar, un segundo tramo más tendido, desde la curva de nivel de los $600 \mathrm{~m}$ hasta el emplazamiento de la población de Casas de Juan Núñez, y, a partir de este punto, un nuevo segmento de mayor pendiente, pero sin que ésta alcance los valores del tramo inferior.

Los perfiles transversales de los tramos superior e inferior muestran claramente las diferencias estructurales y litológicas que se producen a lo largo del recorrido de la rambla. En el superior el cauce se ve fuertemente marcado por el control de la estructura, mientras que en el inferior la propia rambla ha construido su lecho conforme a sus parámetros hidráuli$\cos$, aunque muy mediatizada por los factores antes expuestos.

En conjunto la sinuosidad del cauce es baja, 1.3; sin embargo este índice sube casi a 2.0 en el tramo inferior.

El valor medio de desnivel (12 por 1000) esconde las particularidades del perfil ya señaladas.

\section{V.3. Análisis morfométrico de la rambla de San Lorenzo}

a) Topologia y estructura de la red de drenaje.

La rambla de San Lorenzo es el resultado de la confluencia de tres subsistemas de cauces temporales, los cuales organizan sendas subcuencas. Estos tres aparatos parafluviales nacen en las estribaciones septentrionales de los montes de Chinchilla son de oeste a este: Rambla del Tejar con una superficie de $53.65 \mathrm{~km}^{2}$ supone el $40 \%$ del total de la cuenca de la rambla de San Lorenzo, Rambla de Alatoz o "Barranco del Reventón" $\left(39,22 \mathrm{~km}^{2}\right.$ de cuenca, $30 \%$ de la superficie total) y Barranco de Carcelén ( $38.84 \mathrm{~km}^{2}, 30 \%$ de la cuenca de San Lorenzo). 

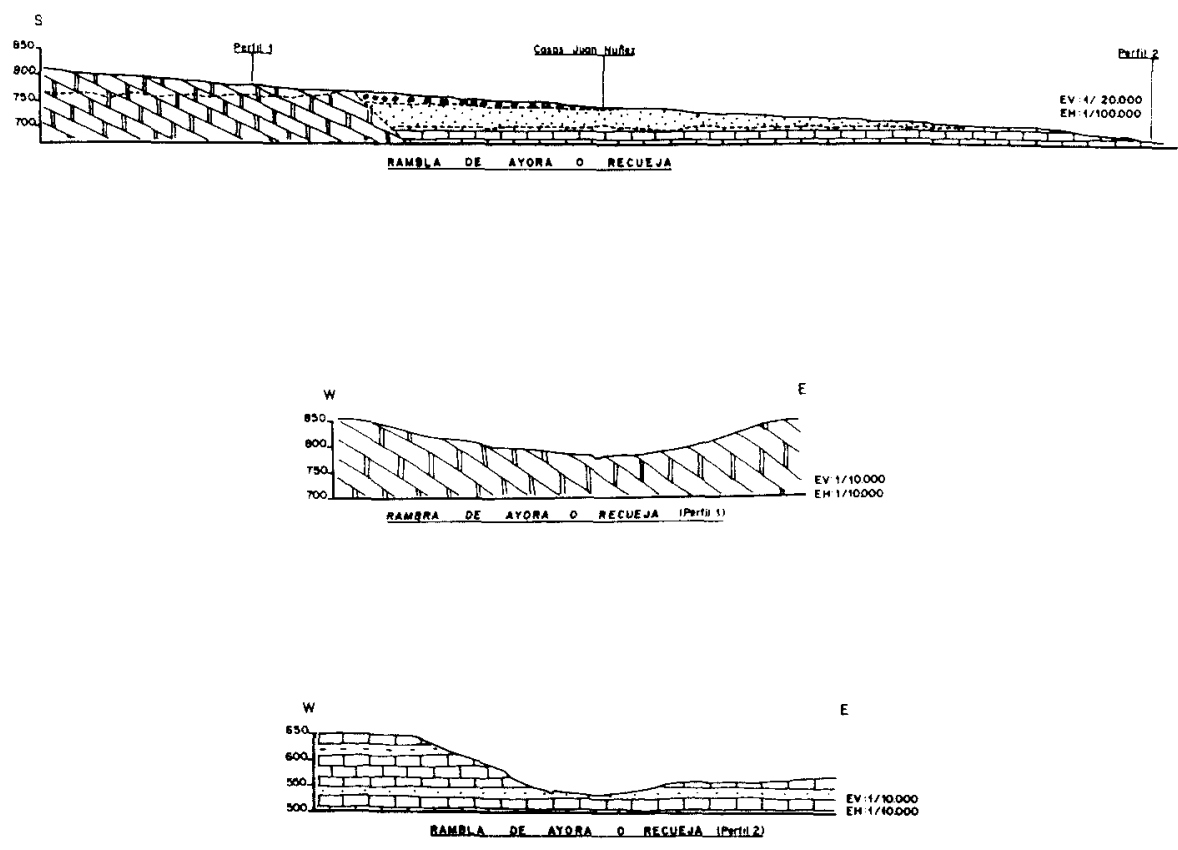

1 1. Dolomias y calizas cretácicas.

2. Cobertera detritica pliocuaternaria.

3. Calizas pliocenas de Alcalá.

Figura 2. Perfil y cortes transversales de la rambla de Ayora. 
Los tres subsistemas discurren genéricamente en dirección SSE-NNW, en función del control estructural de la muela de Carcelén, manteniéndola cuando entran en la fosa del mismo nombre, la cual queda enmarcada al norte por los relieves de La Caballa y el Boquerón que constituyen, a su vez, el borde meridional de la fosa del Júcar. Esta disposición estructural hace que las tres ramblas procedentes de la muela adopten una dirección este-oeste una vez alcanzado el borde septentrional de la fosa, al adaptarse al control tectónico de La Caballa.

La confluencia de la Rambla de Alatoz y el Barranco de Carcelén crea un nuevo canal de orden superior que recibe el nombre de Cañada de la Gitana que a su vez, cuando confluye con la Rambla de El Tejar conforma el canal principal que en el presente trabajo se denomina «Rambla de San Lorenzo". A partir de la citada confluencia, el sentido este-oeste sufre una marcada inflexión y se torna prácticamente $\mathrm{S}-\mathrm{N}$, abriéndose camino ortoclinalmente al eje directriz de las estribaciones más occidentales de La Caballa y entrando en el dominio morfoestructural de al Fosa del Júcar.

b) Características topográficas de la cuenca.

Las mayores alturas de la cuenca superan ligeramente los $1100 \mathrm{~m}$ y se encuentran en diversas muelas situadas al sur. El punto más bajo se corresponde lógicamente con la desembocadura de la rambla de San Lorenzo en el Júcar, que se produce a $520 \mathrm{~m}$. El desnivel máximo es, por lo tanto, de $589 \mathrm{~m}$, siendo el valor más elevado de las ramblas analizadas. La potencialidad erosiva es muy alta, ya que los casi 600 metros de desnivel salvados en los $20 \mathrm{~km}$ que separan en línea recta la divisoria y el eje fluvial se traducen en una pendiente media de 30 metros por 1000 .

A esta potencialidad erosiva derivada de la topografía hay que añadir una elevada densidad de drenaje, que supera el kilómetro por kilómetro cuadrado, y un Índice de Torrencialidad también muy elevado ( 1 canal de primer orden por cada $2.5 \mathrm{~km}^{2}$ de cuenca). No obstante, estas cifras quedan matizadas al analizar las diferentes unidades altimétricas, similares a las expuestas en la rambla de Ayora: un dominio montañoso de elevadas pendientes, un área de transición correspondiente con la fosa de Carcelén y bajos valores de pendiente y por último, el ámbito de fuertes pendientes en la fosa del Júcar.

c) Litología y trazado de la red.

Son tres los ámbitos litológicos que se reparten la cuenca de la rambla de San Lorenzo: el de los materiales dolomíticos y calizos del Cretácico superior, sobre el que se desarrollan las mayores alturas; el de las acumulaciones detríticas cuaternarias, que constituyen el fondo de la fosa de 

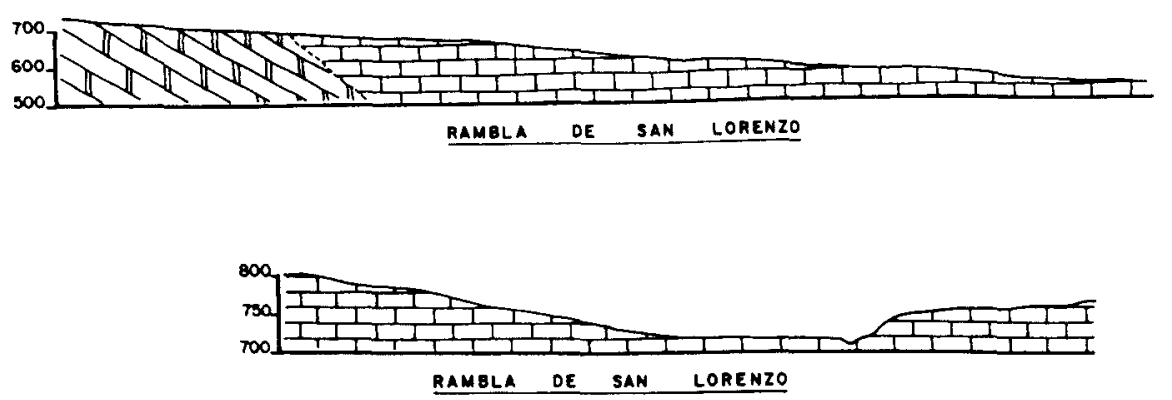

Figura 3. Perfil longitudinal y transversal de la rambla de San Lorenzo.

Carcelén; y el de los conjuntos detríticos y carbonáticos de edad pliocena, aflorantes en el tramo final y más bajo de la cuenca. Estos tres ámbitos se traducen en una acusada heterogeneidad en la naturaleza litológica de los aportes que arrastra la rambla en su lecho. $Y$ a esta diversificación en la aportación de materiales hay que añadir la ya comentada influencia de los caracteres de éstos en el trazado de los cursos.

d) Características del canal principal.

El perfil longitudinal y el transecto (figura 3) muestran como los casi $14 \mathrm{~km}$ del canal principal cortan los tres ámbitos litológicos descritos anteriormente. Los valores de la pendiente varían según el ámbito morfolitológico: 16 por 1000 en el tramo de los estratos cretácicos de las estribaciones occidentales de la Caballa; 12 por 1000 en el área que disecciona el glacis cuaternario; y 20 por 1000 cuando la rambla se encaja en las calizas de Alcalá.

En el perfil se aprecia también con claridad cómo las rupturas de pendiente están muy ligadas a las discontinuidades litológicas. En cualquier caso, el valor medio de la pendiente es relativamente elevado, casi 16 por 1000 , pero, cómo ya se ha visto, es superado en algunos segmentos de la rambla, particularmente en el tramo bajo de su curso.

El índice de sinuosidad se aproxima a 2, adoptando el canal un aspecto tortuoso y meandriforme. Este índice en el tramo más bajo llega a superar el valor 2.5 .

\section{V.4. Análisis morfométrico de la rambla de Alcalá}

La rambla de Alcalá es la más occidental, la que drena una mayor superficie y la más evolucionada del conjunto de ramblas constituido por aquellas que tienen su cabecera en las estribaciones septentrionales de sierra de 
La Caballa. Su estudio se hace de manera detallada, considerándolo como base para la interpretación conjunta de las otras cuatro restantes.

a) Topología y estructura de la red de drenaje.

Los cauces de orden 1 nacen en torno a los $900 \mathrm{~m}$ en la sierra de La Caballa y se van organizando hasta configurar dos canales de orden 2 (Cañizo del Aguilucho y Cañizo del Corral del Cabo), que al unirse generan el canal principal de la rambla de Alcalá (o Cañada de Dominguillo según la toponimia local). Esta rambla, en las proximidades de su desembocadura, recibe un canal secundario de orden 2 , que nace en la misma sierra que los anteriormente citados.

Esta sencilla estructura conforma un sistema de casi $40 \mathrm{~km}$ de cauces, que define una cuenca con marcada densidad de drenaje ( 1.33 $\mathrm{km} / \mathrm{km}^{2}$ ) y acusada torrencialidad (existe un cauce de orden 1 por cada $2.5 \mathrm{~km}^{2}$ de cuenca). La potencialidad erosiva de la red es alta y su trazado está altamente condicionado por la estructura, como lo demuestran los elevados valores de bifurcación de Horton y el trazado rectilíneo del canal principal, que sigue una clara dirección SSE-NNW, coincidente con la de las ramblas que atraviesan la fosa de Carcelén.

b) Características topográficas de la cuenca.

La mayor altura es de $957 \mathrm{~m}$ y el nivel de base se encuentra en la población de Alcalá a $517 \mathrm{~m}$; la diferencia altimétrica es, pues, de $440 \mathrm{~m}$. Este desnivel unido al escaso recorrido entre la cabecera y la confluencia con el río Júcar y a la densidad de la red supone un fuerte riesgo potencial de erosión. En términos generales se pueden distinguir en la cuenca tres ámbitos coincidentes con otros tantos intervalos altitudinales:

- Un ámbito montañoso, cuya altura oscila entre 800 y $957 \mathrm{~m}$, donde nacen los cauces de primer y segundo orden.

- Un ámbito de rampa, (entre 700 y $800 \mathrm{~m}$ de altura), coincidente con los glacis cuaternarios que arrancan de la sierra de La Caballa.

- Un tramo de enlace relativamente brusco, de los 700 a los $517 \mathrm{~m}$, entre la llanura de La Manchuela y el lecho del Júcar.

Al igual que sucedia en las ramblas de San Lorenzo y de Ayora, cada uno de estos tramos altitudinales muestra diferencias de pendientes en los cauces que los surcan.

c) Litología y trazado de la red.

El comportamiento de las diversas unidades litológicas ante la acción modeladora de las aguas esporádicamente canalizadas es similar al ex- 
plicado en el caso de las ramblas anteriores, produciéndose igualmente un fuerte encajamiento en la caliza de Alcalá

d) Características del canal principal.

Los $6.4 \mathrm{Km}$ de la rambla de Alcalá salvan un desnivel de $233 \mathrm{~m}$, lo que supone una pendiente media del 36.4 por 1000 . En el perfil y en los cortes transversales de la figura 4 se pueden apreciar cómo esta pendiente media resulta de tramos claramente diferenciados.

En el tramo inferior de la rambla, cuando ésta se encaja en las calizas de Alcalá, la pendiente llega al 130 por 1000 , valor elevadísimo que indica la torrencialidad y violencia de los caudales cuando circulan por este segmento del canal. Sin embargo, cuando la rambla discurre por los materiales detríticos de la Unidad Superior o de los glacis cuaternarios, la pendiente desciende al 22 por 1000 . El significado geomorfológico de este hecho puede observarse en el perfil 1 de la figura 4 , en el que se constata como en el tramo superior la rambla discurre por la llanura sin haber tenido capacidad de incisión en estos materiales detríticos. El perfil 2 muestra el profundo encajamiento que supone un desnivel de casi 250 metros entre el fondo de la rambla y la llanura culminante. La escasa sinuosidad (1.28) del canal principal, no sufre grandes variaciones a lo largo de su recorrido.
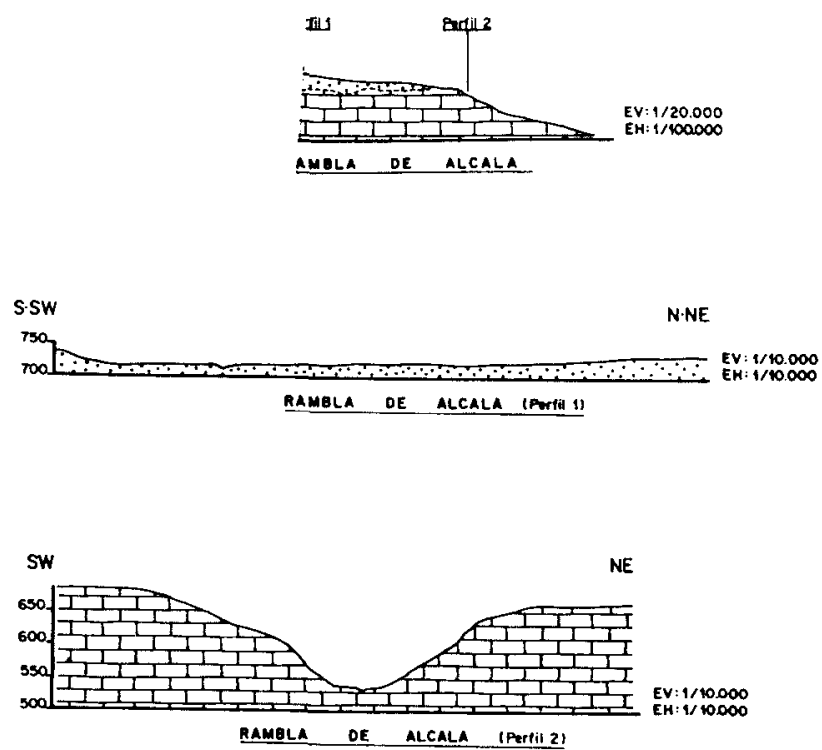

Figura 4. Perfil y cortes transversales de la rambla de Alcalá. 


\section{V.5. Análisis morfométrico de las ramblas de el Cura, Casas del Conde, Mingo y Molinar}

Como se ha indicado, estas cuatro ramblas se analizan conjuntamente siguiendo el modelo interpretativo aplicado a la de Alcalá. El análisis conjunto se justifica por la homogeneidad que presentan, con muchos puntos de coincidencia entre ellas.

a) Lugar de nacimiento y trazado de la red.

Todas las ramblas citadas tienen sus cabeceras en las faldas septentrionales de la sierra de La Caballa. La altura máxima de sus cuencas oscila entre los $944 \mathrm{~m}$ de la rambla del Cura y los 997 de la de Casas del Conde. La red presenta una organización muy simple que no supera el orden 3, e incluso la de Casas del Conde sólo llega al orden 2. El trazado está muy condicionado por factores estructurales y los canales principales siguen una dirección SSE-NNW, que, cómo ya se ha visto, se repite en prácticamente todos los ejes parafluviales afluentes a la hoz del Júcar.

b) Extensión de las cuencas.

A través de los canales principales se drenan pequeñas cuencas cuya extensión fluctúa entre los $6.5 \mathrm{~km}^{2}$ de la rambla de Casas del Conde y los $10.6 \mathrm{~km}^{2}$ del Barranco del Molinar. La única excepción es el Barranco de Mingo, que presenta una cuenca algo mayor: casi $18 \mathrm{~km}^{2}$.

c) Perfiles longitudinales.

Todos los perfiles longitudinales coinciden en mostrar un tramo muy tendido, de pendiente relativamente suave, coincidente con su recorrido por la llanura culminante de La Manchuela. El tramo final de los canales principales presenta, por el contrario, fuerte inclinación coincidiendo con el profundo encajamiento en los materiales de las calizas de Alcalá o en los estratos conglomeráticos de la Facies Puntal Blanco, que en este tramo inferior del Júcar adquieren un importante espesor a medida que se avanza hacia el este. Las pendientes longitudinales medias de los canales presentan valores muy altos y aumentan también progresivamente hacia el este, contrastando los valores próximos a 50 por 1000 de las ramblas del Cura y de Casas del Conde con las fortísimas inclinaciones de los barrancos de Mingo y Molinar, 82 y 91 por 1000 respectivamente (figura 5).

d) Influencia de la litología.

El afloramiento de la Facies Puntal Blanco introduce un factor innovador respecto a las anteriores ramblas. La naturaleza conglomerática de esta facies y su matriz arcillo-limosa facilitan los procesos erosivos y de 
acarcavamiento, comportándose respecto a ellas las capas de caliza de Alcalá como umbrales de resistencia. La Facies Puntal Blanco (F.P.B) forma el lecho rocoso de los canales de las ramblas en su tramo inferior, las cuales se encajan más o menos en él según la potencia estratigráfica: dado que ésta aumenta progresivamente hacia el este, la rambla del Cura se encaja 60 metros en los materiales de la F.P.B, la de Casas del Conde $130 \mathrm{~m}$, el Barranco de Mingo casi 150 metros (desarrollándose todas las márgenes de su canal principal sobre estos materiales) y, por último, el barranco del Molinar se aproxima a los 200 metros. La figura 6 muestra la sucesión de cortes transversales en los tramos bajos de las diferentes ramblas.
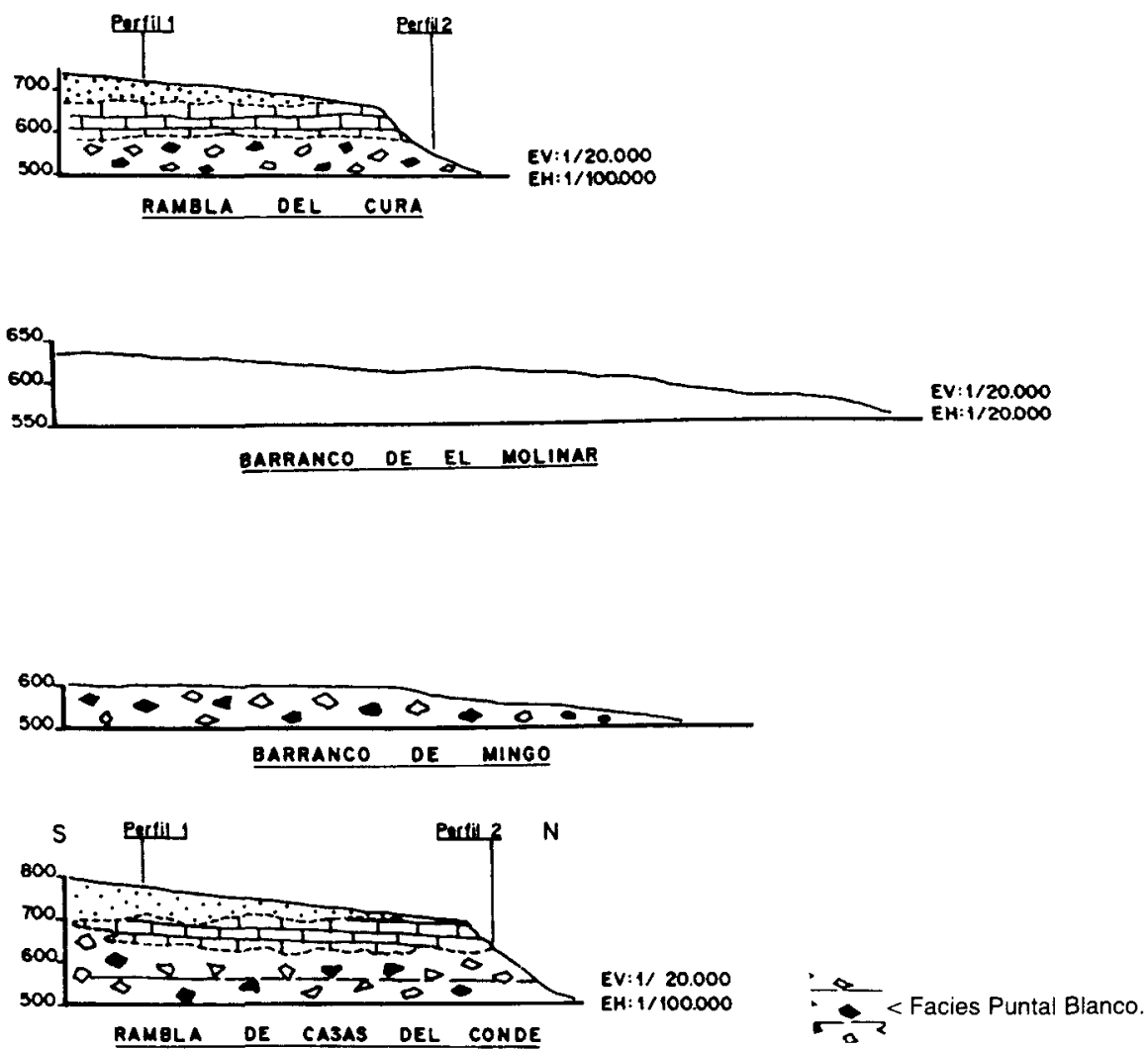

Figura 5. Perfiles longitudinales de las ramblas de el Cura, Casas del Conde, Mingo y Molinar. 
Caracteristicas morfométricas de la red parafluvial de la comarca de la Manchuela...

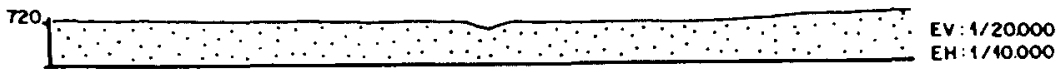

RAMBLA OEL CURA (Perfill)
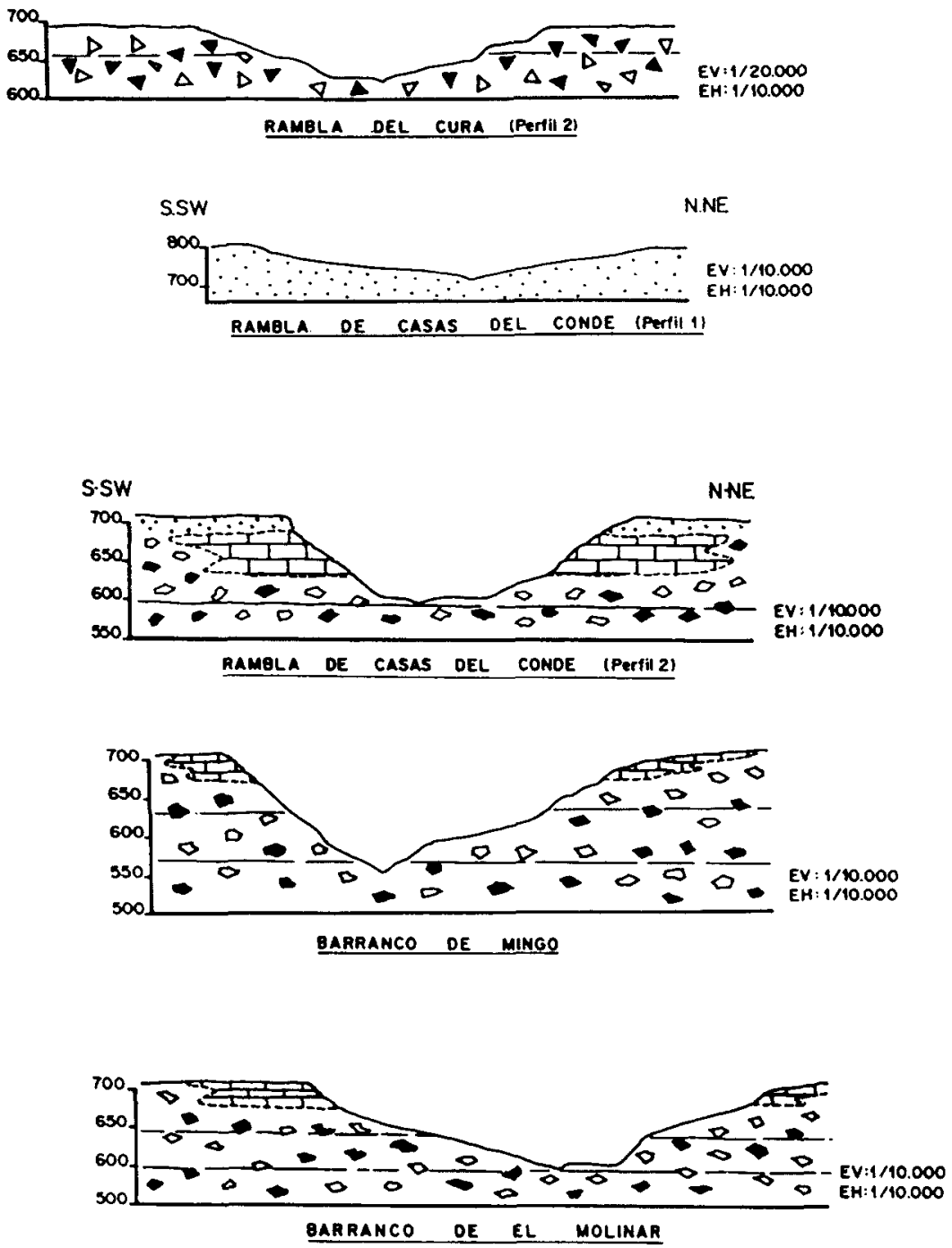

Figura 6. Cortes transversales de los tramos bajos de las ramblas de el Cura, Casas del Conde, Mingo y Molinar. 
La presencia de estos materiales conglomeráticos se traduce paisajísticamente un profundo acarcavamiento de las laderas que enmarcan los fondos de las diferentes ramblas, dando lugar a parajes agrestes y de fuerte potencialidad erosiva.

e) Susceptibilidad a procesos erosivos.

La erosión potencial es elevada tanto por las características del material, según se ha señalado, como por los valores de Densidad de Drenaje e Índice de Torrencialidad. La Densidad de Drenaje en todas las ramblas de este conjunto supera los $2 \mathrm{~km} / \mathrm{km}^{2}$, exceptuando el barranco de Mingo que tiene un valor sensiblemente inferior, $1.5 \mathrm{~km} / \mathrm{km}^{2}$, coincidente con su mayor superficie.

El Índice de Torrencialidad alcanza los valores más altos de todo el sistema parafluvial afluente al Júcar en su tramo de La Manchuela. Concretamente, el Barranco del Molinar presenta la tasa más elevada de torrencialidad, al corresponder $0.70 \mathrm{~km}^{2}$ de cuenca por cada cauce de primer orden. Las restantes ramblas tienen unos valores en torno a $1.5 \mathrm{~km}^{2}$ por cauce de primer orden.

La litología deleznable y la topología de la red explican el alto poder incisivo de estos aparatos parafluviales, en un tramo del valle en el que las precipitaciones son escasas y las cuencas de recepción muy pequeñas. El resultado es que estas pequeñas ramblas han seguido en su encajamiento al lecho del Júcar, en el que tienen su nivel de base, sin que existan vallejos colgados respecto al valle principal. Esta aparente contradicción sólo se entiende por la potencialidad erosiva, que se activa en momentos determinados, ligados a fuertes precipitaciones.

\section{SINNTESIS E INTERPRETACIÓN MORFOMÉTRICA DE LAS RAMBLAS}

Las conclusiones se pueden resumir en los siguientes puntos:

a) Comparativamente con las ramblas del óvalo levantino, las de la Manchuela presentan escasa superficie de cuenca y canales muy cortos. Tan sólo el sistema de la rambla de Abengibre sería comparable a los grandes aparatos levantinos. No obstante, dentro del conjunto de las nueve ramblas estudiadas existe una clara gradación en el tamaño, correspondiendo el más pequeño a las que tienen sus cabeceras en las proximidades de su nivel de base natural, es decir a las que nacen en la sierra de La Caballa.

b) Las alturas máximas de las cuencas son muy modestas, como máximo $1100 \mathrm{~m}$, llegando en el caso de la rambla que nace en la superficie culminante de La Manchuela a no alcanzar los $700 \mathrm{~m}$. Esta precariedad altimétrica, dentro del marco de la Meseta, no impide que los valores medios de la 
pendiente sean muy elevados y que desde este punto de vista las ramblas de La Manchuela superen los valores de algunas grandes ramblas valencianas.

El valor de la pendiente aumenta progresivamente en las ramblas que se ubican en los sectores mas orientales del valle, llegando incluso al 90 por 1000. Este hecho no está motivado por el desnivel altimétrico, el cual es muy semejante en todos los casos (450-550 m), exceptuando los casos extremos de Ayora y San Lorenzo. La razón del aumento de pendiente hay que buscarla en el corto recorrido que tienen las ramblas orientales para salvar desniveles medios en recorridos de poca más de $2 \mathrm{~km}$, mientras que las occidentales, lo hacen con recorridos más largos.

c) La densidad de drenaje y el índice de torrencialidad aumenta de modo progresivo hacia el este, alcanzando los valores más altos en las ramblas que se ubican en este sector. La menor superficie de cuencas y el mayor número de cauces de primer orden, explican las altas densidades de drenaje. El control litológico con un predominio de materiales poco consistentes, justifica la densificación de la red de drenaje.

\section{ANEXO ESTADISTIICO DE LOS VALORES MORFOMÉTRICOS DE LAS RAMBLAS}

Rambla de Abengibre

\begin{tabular}{cccc}
\hline Orden & $N{ }^{\circ}$ de cauces & Kilómetros & Longitud media \\
\hline 1 & 47 & 115 & 2.44 \\
\hline 2 & 12 & 80 & 6.66 \\
\hline 3 & 2 & 10 & 5.0 \\
\hline 4 & 1 & 61 & 61 \\
\hline & 62 & 266 & 4.29 \\
\hline
\end{tabular}

LONGITUD DEL CANAL PRINCIPAL

INDICE DE SINUOSIDAD DEL CANAL PRINCIPAL

ALTURA MÁXIMA DEL CANAL PRINCIPAL

ALTURA MÍNIMA DEL CANAL PRINCIPAL

PENDIENTE MEDIA DEL CANAL PRINCIPAL.

SUPERFICIE DE LA CUENCA

ALTURA MÁXIMA DE LA CUENCA

ALTURA MINIMA DE LA CUENCA

DESNIVEL MÁXIMO DE LA CUENCA

DENSIDAD DE DRENAJE

ÍNDICE DE TORRENCIALIDAD

RAZÓN DE BIFURCACIÓN DE HORTON
$62 \mathrm{Km}$.

1.74

$778 \mathrm{~m}$.

$557 \mathrm{~m}$.

0.0035

$670 \mathrm{~km}^{2}$

$1057 \mathrm{~m}$.

$557 \mathrm{~m}$.

$500 \mathrm{~m}$.

$0.39 \mathrm{k} / \mathrm{km}^{2}$

$1 / 14.22 \mathrm{~km}^{2}$

$\mathrm{N} 1 / \mathrm{N} 2=3.91$

$\mathrm{N} 2 / \mathrm{N} 3=6$

$\mathrm{N} 3 / \mathrm{N} 4=2$ 
Rambla de Ayora o la Recueja

\begin{tabular}{cccc}
\hline Orden & $N{ }^{\circ}$ de cauces & Kilómetros & Longitud media \\
\hline 1 & 54 & 94.25 & 1.74 \\
\hline 2 & 13 & 45.6 & 3.50 \\
\hline 3 & 5 & 35.6 & 7.12 \\
\hline 4 & 1 & 22.5 & 22.50 \\
\hline & 73 & 197.95 & 2.71 \\
\hline
\end{tabular}

LONGITUD DEL CANAL PRINCIPAL

ÍNDICE DE SINUOSIDAD DEL CANAL PRINCIPAL .

$22.5 \mathrm{Km}$.

ALTURA MÁXIMA DEL CANAL PRINCIPAL

1.39

ALTURA MÍNIMA DEL CANAL PRINCIPAL

$812 \mathrm{~m}$.

$535 \mathrm{~m}$.

PENDIENTE MEDIA DEL CANAL PRINCIPAL

0.0123

SUPERFICIE DE LA CUENCA

$191.5 \mathrm{~km}^{2}$

ALTURA MÁXIMA DE LA CUENCA

$1086 \mathrm{~m}$.

ALTURA MINIMA DE LA CUENCA.

$535 \mathrm{~m}$.

DESNIVEL MÁXIMO DE LA CUENCA

$551 \mathrm{~m}$.

DENSIDAD DE DRENAJE

$1.03 \mathrm{k} / \mathrm{km}^{2}$

ÍNDICE DE TORRENCIALIDAD

$1 / 3.54 \mathrm{~km}^{2}$

RAZÓN DE BIFURCACIÓN DE HORTON.

$\mathrm{N} 1 / \mathrm{N} 2=4.15$

$\mathrm{N} 2 / \mathrm{N} 3=2.6$

$\mathrm{N} 3 / \mathrm{N} 4=5$

Rambla de San Lorenzo

\begin{tabular}{cccc}
\hline Orden & $N^{\circ}$ de cauces & Kilómetros & Longitud media \\
\hline 1 & 52 & 84.8 & 1.63 \\
\hline 2 & 13 & 28.2 & 2.17 \\
\hline 3 & 5 & 22.45 & 4.49 \\
\hline 4 & 2 & 8.25 & 4.125 \\
\hline 5 & 1 & 13.825 & 13.825 \\
\hline & 73 & 157.525 & 2.15 \\
\hline
\end{tabular}

LONGITUD DEL CANAL PRINCIPAL

$13.825 \mathrm{Km}$

INDICE DE SINUOSIDAD DEL CANAL PRINCIPAL

1.86

ALTURA MÁXIMA DEL CANAL PRINCIPAL

$736 \mathrm{~m}$.

ALTURA MÍNIMA DEL CANAL PRINCIPAL

$520 \mathrm{~m}$.

PENDIENTE MEDIA DEL CANAL PRINCIPAL

0.0158

SUPERFICIE DE LA CUENCA

$131.71 \mathrm{~km}^{2}$

ALTURA MÁXIMA DE LA CUENCA

$1109 \mathrm{~m}$.

ALTURA MÍNIMA DE LA CUENCA.

$517 \mathrm{~m}$.

DESNIVEL MÁXIMO DE LA CUENCA

$592 \mathrm{~m}$.

DENSIDAD DE DRENAJE

$1.19 \mathrm{~km} / \mathrm{km}^{2}$

INDICE DE TORRENCIALIDAD

$1 / 2.53 \mathrm{~km}^{2}$

RAZÓN DE BIFURCACIÓN DE HORTON

$\mathrm{N} 1 / \mathrm{N} 2=4$

$\mathrm{N} 2 / \mathrm{N} 3=2.6$

$\mathrm{N} 3 / \mathrm{N} 4=2.5$

$\mathrm{N} 4 / \mathrm{N} 5=2.0$ 
Características morfométricas de la red parafluvial de la comarca de la Manchuela...

Rambla de Alcalá

\begin{tabular}{cccc}
\hline Orden & $N^{\circ}$ de cauces & Kilómetros & Longitud media \\
\hline 1 & 12 & 25.2 & 2.1 \\
\hline 2 & 3 & 8.25 & 2.75 \\
\hline 3 & 1 & 6.4 & 6.4 \\
\hline & 16 & 39.85 & 2.49 \\
\hline
\end{tabular}

LONGITUD DEL CANAL PRINCIPAL

ÍNDICE DE SINUOSIDAD DEL CANAL PRINCIPAL

$6.4 \mathrm{Km}$.

ALTURA MÁXIMA DEL CANAL PRINCIPAL

1.28

$750 \mathrm{~m}$.

ALTURA MÍNIMA DEL CANAL PRINCIPAL

$517 \mathrm{~m}$.

PENDIENTE MEDIA DEL CANAL PRINCIPAL

0.0364

SUPERFICIE DE LA CUENCA

$29.77 \mathrm{~km}^{2}$

ALTURA MÁXIMA DE LA CUENCA

$957 \mathrm{~m}$

ALTURA MINIMIMA DE LA CUENCA

$517 \mathrm{~m}$.

DESNIVEL MÁXIMO DE LA CUENCA

$440 \mathrm{~m}$

DENSIDAD DE DRENAJE

$1.33 \mathrm{~km} / \mathrm{km}^{2}$

INDICE DE TORRENCIALIDAD

$1 / 2.48 \mathrm{~km}^{2}$

RAZÓN DE BIFURCACIÓN DE HORTON

$\mathrm{N} 1 / \mathrm{N} 2=4$

$\mathrm{N} 2 / \mathrm{N} 3=3$

Rambla del Cura

\begin{tabular}{cccc}
\hline Orden & $N^{\circ}$ de cauces & Kilómetros & Longitud media \\
\hline 1 & 4 & 8.1 & 2.002 \\
\hline 2 & 2 & 3.5 & 1.750 \\
\hline 3 & 1 & 4.95 & 4.950 \\
\hline & 7 & 16.55 & 2.36 \\
\hline
\end{tabular}

LONGITUD DEL CANAL PRINCIPAL

$4.95 \mathrm{Km}$.

ÍNDICE DE SINUOSIDAD DEL CANAL PRINCIPAL

1.32

ALTURA MÁXIMA DEL CANAL PRINCIPAL

$740 \mathrm{~m}$.

ALTURA MINIMA DEL CANAL PRINCIPAL

$497 \mathrm{~m}$.

PENDIENTE MEDIA DEL CANAL PRINCIPAL

0.0490

SUPERFICIE DE LA CUENCA

$7.52 \mathrm{~km}^{2}$

ALTURA MÁXIMA DE LA CUENCA

$944 \mathrm{~m}$.

ALTURA MINIMA DE LA CUENCA.

$497 \mathrm{~m}$.

DESNIVEL MÁXIMO DE LA CUENCA

$447 \mathrm{~m}$.

DENSIDAD DE DRENAJE

INDICE DE TORRENCIALIDAD

$2.20 \mathrm{k} / \mathrm{km}^{2}$

RAZÓN DE BIFURCACIÓN DE HORTON

$1 / 1.88 \mathrm{~km}^{2}$

$\mathrm{N} 1 / \mathrm{N} 2=2$

$N 2 / N 3=2$ 
Rambla de Casas del Conde

\begin{tabular}{cccc}
\hline Orden & $N^{\circ}$ de cauces & Kilómetros & Longitud media \\
\hline 1 & 4 & 6.3 & 1.575 \\
\hline 2 & 1 & 6.1 & 6.1 \\
\hline & 5 & 12.4 & 2.480 \\
\hline
\end{tabular}

LONGITUD DEL CANAL PRINCIPAL INDICE DE SINUOSIDAD DEL CANAL PRINCIPAL

ALTURA MÁXIMA DEL CANAL PRINCIPAL

ALTURA MÍNIMA DEL CANAL PRINCIPAL

PENDIENTE MEDIA DEL CANAL PRINCIPAL

SUPERFICIE DE LA CUENCA

ALTURA MÁXIMA DE LA CUENCA

ALTURA MINIMA DE LA CUENCA

DESNIVEL MÁXIMO DE LA CUENCA

DENSIDAD DE DRENAJE

ÍNDICE DE TORRENCIALIDAD

RAZÓN DE BIFURCACIÓN DE HORTON
$6.1 \mathrm{Km}$.

1.24

$790 \mathrm{~m}$.

$490 \mathrm{~m}$

0.0491

$6.50 \mathrm{~km}^{2}$

$997 \mathrm{~m}$.

$490 \mathrm{~m}$.

$507 \mathrm{~m}$.

$1.90 \mathrm{k} / \mathrm{km}^{2}$

$1 / 1.5 \mathrm{~km}^{2}$

$\mathrm{N} 1 / \mathrm{N} 2=4$

Rambla de Mingo

\begin{tabular}{cccc}
\hline Orden & $N^{\circ}$ de cauces & Kilómetros & Longitud media \\
\hline 1 & 13 & 20.6 & 1.58 \\
\hline 2 & 2 & 5.9 & 2.95 \\
\hline 3 & 1 & 1.5 & 1.5 \\
\hline & 16 & 28 & 1.75 \\
\hline
\end{tabular}

LONGITUD DEL CANAL PRINCIPAL

$1.5 \mathrm{Km}$.

INDICE DE SINUOSIDAD DEL CANAL PRINCIPAL ...............

ALTURA MÁXIMA DEL CANAL PRINCIPAL ......................... $607 \mathrm{~m}$.

ALTURA MÍNIMA DEL CANAL PRINCIPAL .......................... $483 \mathrm{~m}$.

PENDIENTE MEDIA DEL CANAL PRINCIPAL....................... 0.0826

SUPERFICIE DE LA CUENCA

$17.91 \mathrm{~km}^{2}$

ALTURA MÁXIMA DE LA CUENCA

$953 \mathrm{~m}$.

$480 \mathrm{~m}$.

$473 \mathrm{~m}$.

DESNIVEL MÁXIMO DE LA CUENCA

$1.56 \mathrm{k} / \mathrm{km}^{2}$

DENSIDAD DE DRENAJE

$1 / 1.37 \mathrm{~km}^{2}$

ÍNDICE DE TORRENCIALIDAD

$\mathrm{N} 1 / \mathrm{N} 2=6.5$

$\mathrm{N} 2 / \mathrm{N} 3=2$ 
Características morfométricas de la red parafluvial de la comarca de la Manchuela...

Rambla de El Molinar

\begin{tabular}{cccc}
\hline Orden & $N^{\circ}$ de cauces & Kilómetros & Longitud media \\
\hline 1 & 15 & 13 & 0.86 \\
\hline 2 & 5 & 6.6 & 1.32 \\
\hline 3 & 1 & 2 & 2 \\
\hline & 21 & 21.6 & 1.08 \\
\hline
\end{tabular}

LONGITUD DEL CANAL PRINCIPAL

2

ÍNDICE DE SINUOSIDAD DEL CANAL PRINCIPAL ................ 1.14

ALTURA MÁXIMA DEL CANAL PRINCIPAL ........................ $663 \mathrm{~m}$.

ALTURA MINIMA DEL CANAL PRINCIPAL ........................ $480 \mathrm{~m}$.

PENDIENTE MEDIA DEL CANAL PRINCIPAL...................... 0.0915

SUPERFICIE DE LA CUENCA ...................................... $10.625 \mathrm{~km}^{2}$

ALTURA MÁXIMA DE LA CUENCA ….............................. $954 \mathrm{~m}$.

ALTURA MINIMA DE LA CUENCA ................................... $480 \mathrm{~m}$.

DESNIVEL MÁXIMO DE LA CUENCA ….......................... $474 \mathrm{~m}$.

DENSIDAD DE DRENAJE ....................................... $2.03 \mathrm{k} / \mathrm{km}^{2}$

ÍNDICE DE TORRENCIALIDAD .................................. $1 / 0.70 \mathrm{~km}^{2}$

RAZÓN DE BIFURCACIÓN DE HORTON......................... N1/N2=3

$\mathrm{N} 2 / \mathrm{N} 3=5$

\section{BIBLIOGRAFIA}

Camarasa Belmonte, A.M. (1995): Génesis de crecidas en pequeñas cuencas semiáridas. Ministerio de Obras Públicas, Transportes y Medio Ambiente. Madrid. 252 págs.

Fernandez Fernandez, A. (1996): Geomorfología del cañón del río Júcar en la comarca de la Manchuela (Albacete). Universidad Complutense. Madrid. 721 págs.

Mateu Belles, J.F. (1974): "La rambla de la Viuda. Clima e hidrologia". Cuadernos de Geografía. Págs 47-68.

MATEu Belles, J.F. (1989): “Ríos y ramblas mediterráneos. Avenidas fluviales e inundaciones en la cuenca del Mediterráneo". Instituto Universitario de Geografía. Alicante. 150 págs. 